\title{
General Psychiatry Viral vectors as a novel tool for clinical and neuropsychiatric research applications
}

\author{
Yao Wang, ${ }^{1,2}$ Zhiwei Hu, ${ }^{2}$ Peijun Ju, ${ }^{3}$ Shan Yin, ${ }^{1,2}$ Fujie Wang, ${ }^{1,2}$ Oudong Pan, ${ }^{2}$ \\ Jinghong Chen ${ }^{3}$
}

To cite: Wang Y, Hu Z, Ju P, et al. Viral vectors as a novel tool for clinical and neuropsychiatric research applications. General Psychiatry 2018;31:e000015. doi:10.1136/ gpsych-2018-000015

Received 15 August 2018 Revised 12 September 2018 Accepted 12 September 2018
Check for updates

C Author(s) (or their employer(s)) 2018. Re-use permitted under CC BY-NC. No commercial re-use. See rights and permissions. Published by BMJ.

${ }^{1}$ Research and development department, OBi0 Technology (Shanghai) Corp., Ltd., Shanghai, China

${ }^{2}$ Marketing department, OBiO Technology (Shanghai) Corp., Ltd., Shanghai, China

${ }^{3}$ Shanghai Mental Health Center, Shanghai Jiao Tong University School of Medicine, Shanghai Key Laboratory of Psychotic Disorders, Shanghai, China

Correspondence to YaoWang; wyao@obiosh.com and Jinghong Chen; chenjh_008@hotmail.com

\section{ABSTRACT}

Background A viral vector is a genetically modified vector produced by genetic engineering. As pathogenic genes in the virus are completely or largely eliminated, it is safe to be widely used in multidisciplinary research fields for expressing genes, such as neuroscience, metabolism, oncology and so on. Neuroscience and psychiatry are the most closely related disciplines in either basic research or clinical research, but the application of viral vectors in neuropsychiatry has not received much attention or not been widely accepted.

Objective This article will focus on the application of viral vectors in basic and clinical neuropsychiatric research. Methods By using viral vectors, scientists can perform neurological labelling, gene expression regulation and physiological manipulation for investigating phenomenon from molecular mechanisms to behaviours. At the same time, to treat mental or neurological disorders, viral vectors can be designed for gene therapy, which alter gene expression levels or repair mutated genes in the brains of patients.

Perspective Viral vectors play an important role in basic research and clinical applications. To further understand brain function and prevent mental and neurological diseases, we hypothesize that viral vectors could be used along with various advanced technologies, such as sequencing and high-throughput expression analysis in the neuroscience research field.

\section{BACKGROUND}

The human brains, one the most complex structure in the world, is considered as the origins of thoughts, emotions and behaviours. It purportedly contains $8.6 \times 10^{10}$ neurons $^{1-3}$ and $8.5 \times 10^{10}$ glial cells. ${ }^{2}$ Neurons interact combinatorically through the $10^{3}-$ $10^{4}$ synapses, and they formed links with each other. ${ }^{34}$ Hence, the development and application of new tools is urgent needed to understand the connexion and function between neuron and glia. Recently, we have been able to have a better understanding of the basic principles of the neural system and brain function owing to the development of viral vector systems, molecular biology techniques and optogenetic techniques. ${ }^{5-7}$ Nevertheless, viral vector systems are not widely applied in basic research and clinical therapy. In view of this, we would like to introduce the application of viral vector systems in neuropsychiatric research.

\section{APPLICATION OF VIRAL VECTOR IN NEUROPSYCHIATRY RESEARCH}

Following the Human Genome Project, the goal of studying pathogenic mechanisms and developing effective diagnostic as well as therapeutic approaches for brain disorders has become 'the final frontier' and as a result, an in-depth understanding of the brain research boom has been launched on a global scale. ${ }^{9}$ Three universal goals of neuroscience are to understand, to protect and to mimic the brain. Since neuropsychiatry belongs to neuroscience, we would like to apply the viral vector techniques under the guidance of neuroscience principals. The gene studies, protein functions and neuronal circuits' activity together with behavioural tests would unravel the gene functional verification and disease pathophysiology. We aim to verify genetic risk factors at the molecular level and decipher the neuronal cellular activity and the development of neural circuits' biomarkers. To achieve these goals, the combination of multiple technologies has become indispensable to research ${ }^{10}$ table 1 . Interestingly, viral vectors play an important role in these technologies.

\section{Introduction and experimental selection of viral vectors}

A virus is a non-cellular form composed of nucleic acid molecules and proteins that replicates only inside the living cells of other organisms, whereas the viral vector is a modified gene-carrying tool. Since 1972, when Paul Berg first engineered the SV40 virus and successfully infected monkey kidney culture cells, ${ }^{11}$ a large number of viral vectors have 
Table 1 Features of technical approaches commonly used for studying neuroscience

\begin{tabular}{|c|c|c|c|c|c|}
\hline \multirow{2}{*}{ Main technlogy } & \multirow[b]{2}{*}{ Tools } & \multirow[b]{2}{*}{ Acute onset } & \multicolumn{3}{|l|}{ Features } \\
\hline & & & Behaving animals & Cell-type specific & Protein specific \\
\hline Optogenetic & ChR2, NpHR, oCHIEF & $\sqrt{ }$ & $\sqrt{ }$ & $\sqrt{ }$ & \\
\hline Calcium imaging & GCaMP, RCaMP, CAMPARI & $\sqrt{ }$ & $\sqrt{ }$ & $\sqrt{ }$ & \\
\hline $\begin{array}{l}\text { Genetic } \\
\text { manipulation }\end{array}$ & $\begin{array}{l}\text { Overexpression, knock-out, } \\
\text { knock-down, CRISPR }\end{array}$ & & $\sqrt{ }$ & $\sqrt{ }$ & $\sqrt{ }$ \\
\hline \multirow[t]{3}{*}{ Pharmacology } & Traditional pharmacology & $\sqrt{ }$ & $\sqrt{ }$ & & $\sqrt{ }$ \\
\hline & Pharmacogenetics (DREADDs) & $\sqrt{ }$ & $\sqrt{ }$ & $\sqrt{ }$ & \\
\hline & Genetically -encoded toxin & & $\sqrt{ }$ & $\sqrt{ }$ & $\sqrt{ }$ \\
\hline
\end{tabular}

CRISPR, Clustered regularly interspaced short palindromic repeats; CaMPARI, Calcium-modulated photoactivatable ratiometric integrator; ChR2, Channelrhodopsin-2; DREADD, Designer receptors exclusively activated by a designer drug; NpHR, Halorhodopsin.

been developed for research and clinical therapy. They are characterised by high safety, low immunogenicity and a marker-containing gene, and are often used for in vivo or in vitro environment for gene expression manipulation in cell. So far, viral vectors which commonly used in laboratories include adeno-associated virus (AAV), lentivirus, adenovirus and retrovirus. These four viral vectors have distinct characteristics, among which AAV has been extensively developed and applied in neuroscience and gene therapy. AAV has high gene delivery ability throughout most human tissues, low immunogenicity and capsid protein diversity. ${ }^{12}$

With viral vectors, we are able to achieve the following four important research goals in the brain (figure 1). The first is the location specificity of gene expression with stereotactic injection technology. The second is the time specificity, that is, injection of viral vectors at specific times, or by molecular biology methods, such as the application of the TRE promoter, to express genes at a specific time. The third is the cell-type specificity of gene expression by a specific promoter, which provides us with a fine interpretation of brain function. The fouth is the experimental task. For neuroscience research, there are several important tasks including the labelling of neurons, neural circuit and brain regions, the expressing of genes and the manipulating and observing of the neurons in brain at the physiological level (figure 1).

\section{Viral vectors as a tool for cell labelling}

Neural labelling and tracing technology is the basis of neuroscience research. Traditional anatomical techniques have met difficulty in meeting the needs of neurological research. These requirements for research are mainly concentrated in the following areas: greater precision, such as subcellular labelling; more specificity, such as labelling of an activity-related cell ensemble ${ }^{14-16}$; labelling at the physiological level rather than ex vivo. To achieve these goals, a variety of viral vectors and novel

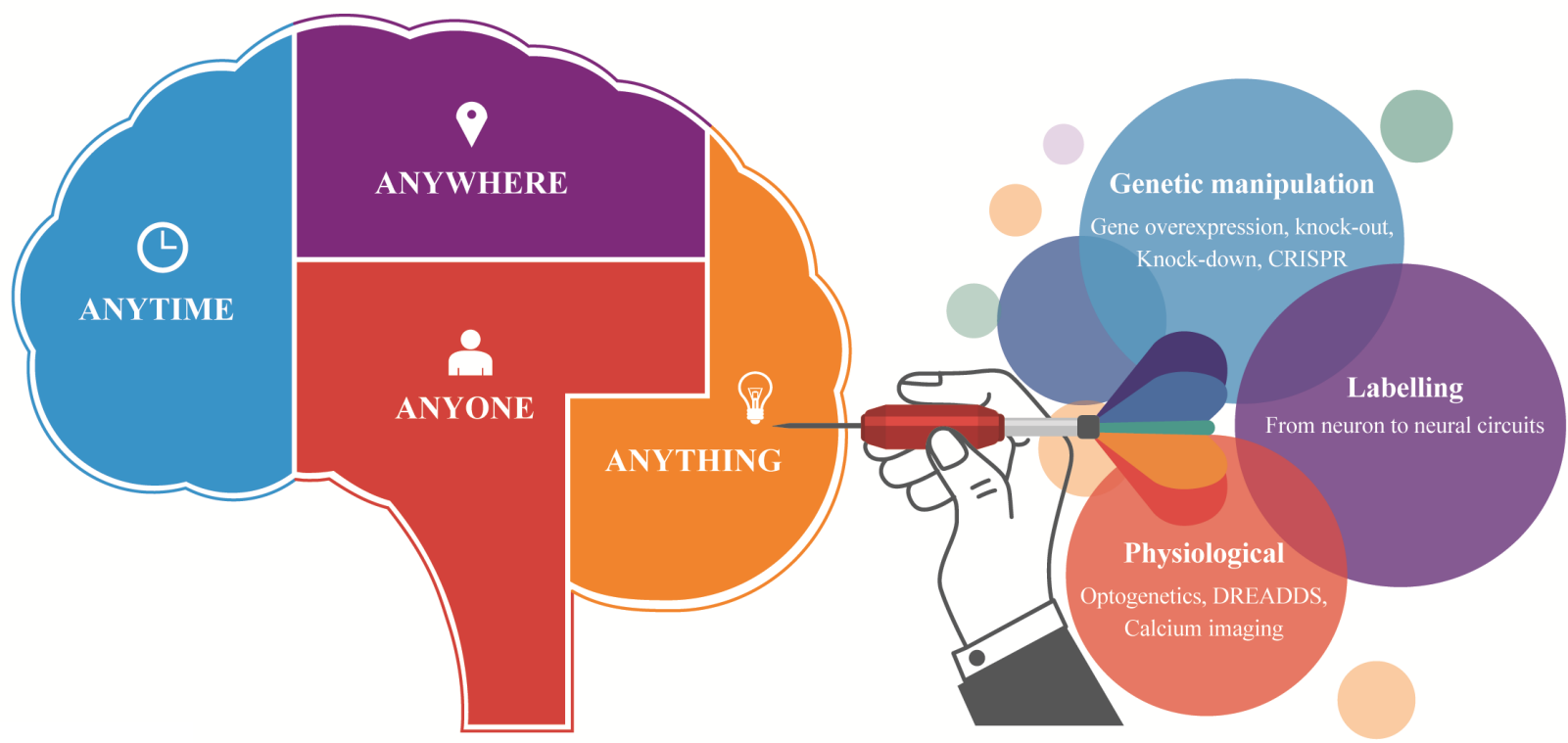

Figure 1 Application of viral vectors in neuroscience research. 
fluorescent labelling tools have been developed, ${ }^{17-19}$ such as the fluorescent protein FT-Slow, ${ }^{20}$ in which colour can be transferred within a time-dependent manner; and the GRASP system, in which the GFP protein is split into two parts for labelling synaptic connexions. ${ }^{21-23}$

\section{Cell type-specific labelling}

With specific promoters, such as hSyn, ${ }^{24}$ CaMKIIa ${ }^{25}$ and the GFAP promoter, ${ }^{26}$ we can label and trace a population of cells, including mature neurons, projection neurons and astrocytes at the cellular level with fluorescent proteins. In addition, we also could obtain the cell type-specific gene expression in transgenic animals ${ }^{27}$ with the cre-dependent strategy. ${ }^{28}{ }^{29}$ This strategy was very effective for labelling cells that are difficult to calibrate by specific promoters, including dopaminergic neurons, microglia ${ }^{30}$ and so forth. To label immature cells in the central nervous system, including neural stem cells and glia precursor cells, we could also use a retrovirus ${ }^{31} 32$ to infect the immature cells or use the nestin-cre line mice ${ }^{33}$ in combination with cre-dependent expression virus.

Cutting-edge research has begun to focus on neuronal labelling and manipulation at the subcellular level. The principle of this method lies in signal-specific gene-directed expression. Common signal peptides include the nucleus signal peptide NLS, ${ }^{34}$ transmembrane signal peptide TM, ${ }^{21}$ mitochondrial signal peptide ${ }^{35}$ and cell body signal peptide $\mathrm{ST} .{ }^{36}$ Based on signal peptides, various tools have been developed, such as the light-controllable CRY2/CIB system locating PSD (post-synaptic density), which could manipulate postsynaptic receptor activities ${ }^{37}$; and GRASP technology ${ }^{2122}$ and enhanced Dual-eGRASP technology ${ }^{34}$ for synaptic connexion labelling. These novel techniques provided better ideas for elucidating the precise functions of the nervous system.

Based on the characteristics of the TRE promoter, we can control gene expression at a specific time. The initiation of the TRE promoter depends on the binding of tTA or rtTA. Doxycycline (Dox) can bind to tTA or rtTA, respectively, blocking (Tet-off system) and promoting (Tet-on system) TRE promoter activation, so researchers can determine the timing of expression of the TRE and its genes by controlling the time of delivering Dox to the animal. ${ }^{38}$ On this basis, we can also specifically label the cell ensemble, a group of cells that respond to a specific task. In recent years, Liu and colleagues used the immediate early gene c-fos in combination with TRE promoter and Dox to label memory-related cell clusters (Engram cells). ${ }^{16}$ In July of 2017, Hyung-Bae Kwon and Alice Y Ting used light as a switch to construct a more precise and new activity-related cell clustering technology, which relies on the upregulation of intracellular $\mathrm{Ca}^{2+}$ concentration driven by neuronal activity as a signal. ${ }^{14} 15$

\section{Labelling methods for neural circuits}

Understanding complex connexions and modifications of neural circuits is critical for revealing the pathogenesis of disease or developing new therapies. Traditional neural tracing methods, sucg as electrom microscopy, Golgi staining, and dyes, can show the morphology and projection of neurons. Furthermore, a few tracers such as plant lectin and fluorescent gold can achieve trans-synaptic labelling. However, these methods have the disadvantages giving an indirect signal, non-specific orientation and severe attenuation across the postsynaptic signal. Importantly, these dyes cannot carry genes. Since Kristensson first applied a herpes simplex virus (HSV) to trans-synaptic label neural circuits, ${ }^{39}$ the application of viral vectors in tracing neural circuits has received increasing attention.

There are two fundamental problems regarding neural circuits labelled by a virus. Clear labelling directions would be required (forward: soma-to-axon; reverse: axonto-soma) and the other one would be cross-synaptic or not. A neurotropic virus is a type of virus with autonomous replication ability, so it can rapidly replicate after infecting a primary neuron and infects another neurons connected to it to achieve trans-synaptic purposes (e,g, rabies virus (RV) and herpers simplex virus (HSV)). However, neurotropic viruses are highly pathogenic and infectious, and are more suitable for the analysis of unknown neural pathways. They are not suitable for studying general physiological and pathological processes. Conversely, AAV is increasingly used for neural circuit tracking because of its lack of replication ability and extremely low immunogenicity.

Conventional AAV vectors cannot cross the synapses. Hence, improved viral anterograde and retrograde tracing has become an important neuroanatomical tract-tracing tool for the characterisation of neuronal networks. Neuronal axons can be labelled through tracking fluorescent proteins expressed by viral vectors, ${ }^{40}$ (i.e.anterograde tracing). Anterograde tracing is often used to label known downstream brain regions, and retrograde tracking is required if we need to find the upstream brain regions. Both fluorescent dyes and viral vectors have the capability of retrograde tracking. However, fluorescent dyes are unable to carry genes. Likewise, neurotropic viruses are highly immunogenic and are not ideal reverse-tracking tools either. Strikingly, in 2016, Karpova's group first constructed the AAV vector, rAAV2-retro serotype vector, with efficient reverse tracing ability, ${ }^{41}$ which could be absorbed at the axon end and retrograde to the nucleus to express fluorescent protein along the cytoskeleton (figure 2A).

In addition, there have been efforts to perform antero-trans-synaptic or retro-trans-synaptic labelling with AAV vectors in recent years. As in 2016, Zingg and colleagues found that high-titre AAV2/1 serotype viral vectors can achieve a trans-synaptic labelling. ${ }^{42}$ In 2013, Xu and colleagues constructed a WGA-cre-based trans-synaptic marker manipulation system. ${ }^{43}$ These non-synaptic or trans-synaptic operations have great benefit for the specific study of neural circuits. ${ }^{28}$ 
A

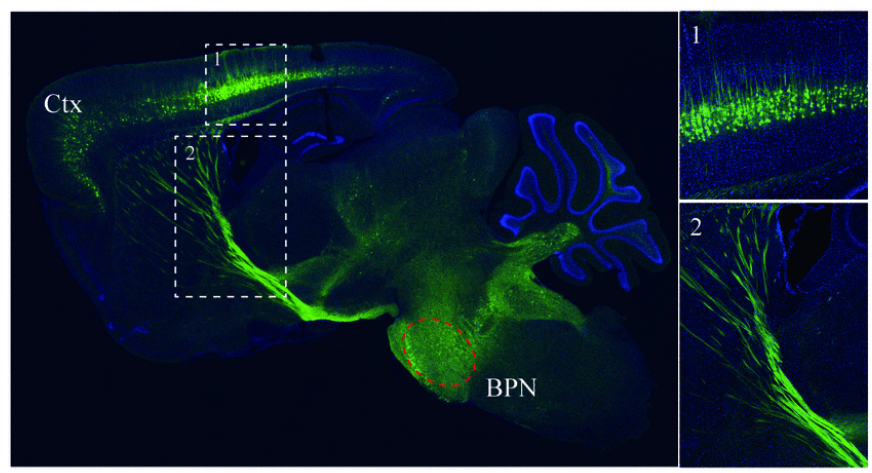

B

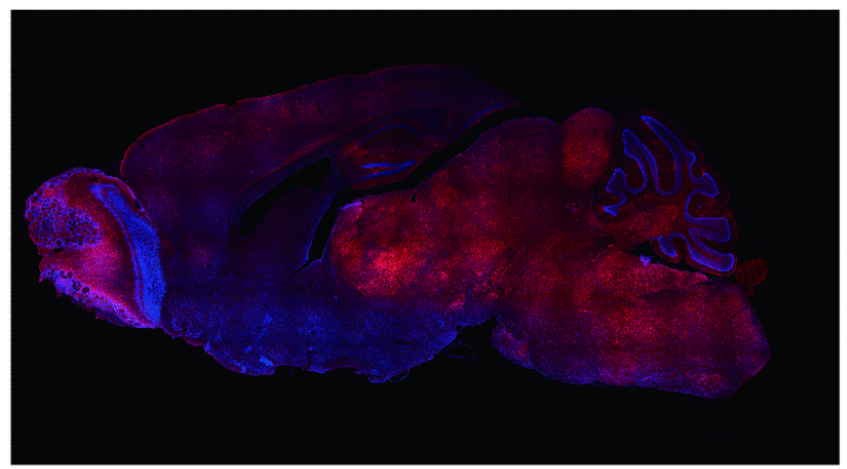

Figure 2 Novel adeno-associated virus (AAV) viral vector mediated non-trans-synaptic retrograde tracing and trans-bloodbrain barrier labelling. (A) By injecting rAAV2-retro adeno-associated virus into the basal pons, the cortical cerebral tract pathway is projected by the reverse-labelled cortex. (B) The whole brain gene expression is obtained by injecting a trans-bloodbrain barrier associated viral vector in the tail vein of the rat.

\section{Cross blood-brain barrier with AAV to infect the whole brain}

We used to deliver viral vectors to specific brain regions and express specific genes or fluorescence with the brain stereotactic injection technique. However, when we require extensive labelling or a gene expression of interest in multiple brain regions, traditional methods of localised injection are not suitable due to limited virus infection efficiency. Deverman's group constructed a series of viral vector AAV-PHP.B and AAV-PHP.eB serotypes $^{44}$ that were able to cross the blood-brain barrier. We only need to inject a proper amount of AAV vector into the tail vein. Gene expression was observed in most areas of the brain (figure 2B). Using this vector, a large amount of research and gene therapy methods can be improved upon. If combined with specific expression elements, the vector is promising as a model for precise, non-invasive treatment of genetic diseases in the brain.

\section{Genetic manipulation method based on viral vectors}

To overexpress genes in the brain, it is necessary to select different promoters and vector types according to different types of neurons. The target genes were then inserted into the viral vector, followed by infecting the cells along with the viral vector. Any gene, such as neurotrophic factors or toxic factors (DTA, etc), could be overexpressed. ${ }^{33}$ The most important thing is to make sure that viral vectors have enough capacity to package the expressed genes and the expressed genes do not affect the normal physiological state of the virus.

Techniques for downregulating expression levels of genes include gene knockout and gene knockdown methods. The constructs of traditionally conditional knockout mice ${ }^{27}$ have the disadvantages of a long construction period, high cost, poor regional specificity and doubtful developmental compensation, although this technique can establish a high-efficiency gene knockout system. At present, we can obtain specific gene knockout by injecting the AAV viral vector containing cre recombinase into the specific brain region of flox mice, thereby achieving higher gene knockout region specificity and eliminating the problem of gene function compensation caused by development. In addition, the gene knockout efficiency by virus injection is faster and less costly, which is favoured by researchers. ${ }^{45}{ }^{46}$ In addition to gene knockout, gene knockdown based on RNA interference technology is also a well-known method for gene expression downregulation. For that purpose, we need to package RNA interference sequences (siRNA or shRNA) into viruses and to obtain gene knockdown of cells in specific regions by means of stereotactic injection. ${ }^{38} 47$

In addition to the above techniques, the emerging ZFN, TALEN and CRISPR/Cas $9^{48} 49$ gene editing technologies are new technologies for genomic modification. The principles of these techniques are to induce genomic double-stranded DNA breaks at specific loci. The target gene is then engineered by non-homologous end joining or homologous recombination. Due to the characteristics of easy construction, low cost and high efficiency of CRISPR/Cas9, it has been the most widely used technology for generating gene knockout, knock-in models. For the purposes of knocking out a gene, we can use viral vectors as delivery devices for carrying Cas9 protein and sgRNA to cause double-strand breaks in DNA. ${ }^{50}$ This strategy could help us with screening mutant genes and thereby finding key pathogenic genes for neuropsychological diseases such as autism, depression and obsessive-compulsive disorder. For the purpose of knocking-in or replacing a gene, it is also necessary to use the virus to express donor DNA as a homologous recombination repair template to repair the gene mutation site, apart from carrying the above Cas 9 protein and sgRNA. ${ }^{51}$ Therapeutic applications of the CRISPR/Cas9 system in gene therapy have provided a new tool for diseases caused by gene mutation. Moreover, a dCas9-based endogenous gene transcriptional activation or inhibition technique based on the mutant Cas9 protein-dCas9 protein, which lacks the ability to cleave DNA, has been developed recently. In this way, we can bind dCas9 protein to specific sgRNA and transcriptional activation (such as VP64) 
or inhibitory elements (such as KRAB), which anchor upstream of the transcription start point. We thereby perform overexpression or knockdown of specific endogenous genes. The method has brought unprecedented convenience for overexpressing large fragment genes and polytopic transmembrane proteins and laid the foundation for the improvement of endogenous genes. ${ }^{52}{ }^{53} \mathrm{In}$ summary, with the help of viral vectors and various gene manipulation technologies, we are able to screen genes related to neurological diseases, study the function of risk genes for disease and develop gene therapy.

\section{Viral vectors mediated physiological manipulation and observational techniques}

The manipulation and observation of neurons or neuronal circuit activity helps in understanding of behavioural changes and the underlying neural mechanisms. Classical studies often use electrophysiological techniques to record or stimulate neurons while behavioural changes were examined. In the past decade, the development of novel technologies such as optogenetics, chemical genetics and calcium imaging has further facilitated this research, and viral vectors play a key role in the application of these new technologies.

\section{Application of optogenetics}

In the past 10 years, ${ }^{54}$ optogenetics has become one of the most important technologies invented in the field of neuroscience. The principle of optogenetics is that the engineering neurons expressed exogenous photosensitive proteins in specific cell types, and can precisely control the activity of nerves or glial cells with light. The results from optogenetics were supplemented by behavioural experiments for interpreting the neurons or neural circuits. The accuracy of optogenetics can achieve the millisecond level in time, and the spatial precision can reach the level of single cell or even organelle. Compared with the traditional electrophysiological stimulation of cells, specific cells can be activated and inhibited by optogenetic technology.

The principle of optogenetics relies on genetic modification of light-gated ion channels. The most commonly used light-sensitive channel for activating neuronal activity is ChR2, which is a non-selective cation channel. When ChR2 absorbs blue light, channels are open with cation influx, and depolarisationi is caused. Eventually, the process could control the electrical excitability of neurons. Similarly, NpHR channel is a commonly used channel to inhibit neuronal activity on account of the influx of chloride ions after light stimulation, and neuronal activity is inhibited. A variety of genetically modified light-sensitive channels based on the original ChR2 and NpHR were constructed. For example, ChR2 (H134R) has a stronger photocurrent but a slower opening. ChR2 (C128S/D156A) has hypersensitive channel with yellow-green light-off and blue-green light-on. ChETAs or oCHIEF channels responded to high-frequency light stimulation..$^{55}$ eNpHR3.0 channel has a short response time and responsive reaction.

In addition to the behavioural studies only with viral vectors that directly express light-sensitive channels, ${ }^{25}$ we can also combine light-sensitive channels with specific promoters or Cre-dependent expression strategies. In this way, we could get more accurate application of light-sensitive channels to trace specific cells or specific neural circuits. ${ }^{30}$ In addition, with the help of mutant light-sensitive channels in response to high-frequency stimuli, such as oCHIEF channels, we have achieved plasticity enhancement (LTP) and inhibition (LTD) that induce long-term synaptic transmission in specific neural circuits for studying the relationship of synaptic transmission plasticity with behaviour. ${ }^{56}$ A large number of studies have also used optogenetics in tissues and organs other than the brain, such as the use of optogenetic techniques to control the frequency of heart beats, ${ }^{57}$ and regulate blood glucose by controlling the synthesis of insulin in engineered cells. ${ }^{58}$

Based on basic research, researchers aimed to develop new treatment options for neurological diseases by combining optogenetics with a variety of other technologies. In the treatment of epilepsy, we can collect the EEG signal to figure out the brain region where epilepsy occurs, and then embed the fibre in the region to express the light-sensitive channel. After the symbolic EEG signal of epilepsy is detected, the fibre transmitted light stimulation and inhibition so that inhibition of epilepsy could happen (figure 3A). For Parkinson's disease, deep brain stimulation (DBS) is commonly used in clinical practice. Moreover, $60 \mathrm{~Hz}$ DBS can alleviate gait and language problems, which cannot alleviate tremors; on the contrary, high frequency $(>100 \mathrm{~Hz})$ DBS can relieve tremors. Therefore, combined with optogenetic techniques (figure 3B), we will be able to simultaneously give animals two types of stimuli to alleviate motor control disorders. ${ }^{59}$ In the field of drug addiction research, we could use optogenetic techniques to induce LTD for changing addiction behaviour in the addiction-related brain regions by means of low-frequency light stimulation ${ }^{60}$ (figure 3C). In the future, the unique high temporal and spatial resolution characteristics of optogenetics technology will continue to develop, combined with the neuronal and neural circuit-specific expression technology of viral vectors, which will further promote the development of research into neurological diseases.

\section{Application of chemical genetics technology}

Chemical genetics (or pharmacological genetics) is another important technology that has been developed rapidly in recent years. It can be artificially controlled by engineering G-protein coupled receptors so that they can only bind to artificial small molecule ligands to regulate receptor activity. The most commonly used chemical genetics system is the DREADDs technology, which was developed by Sternson and Roth. ${ }^{61}$ By changing the structure of the acetylcholine receptor, they can only bind to 

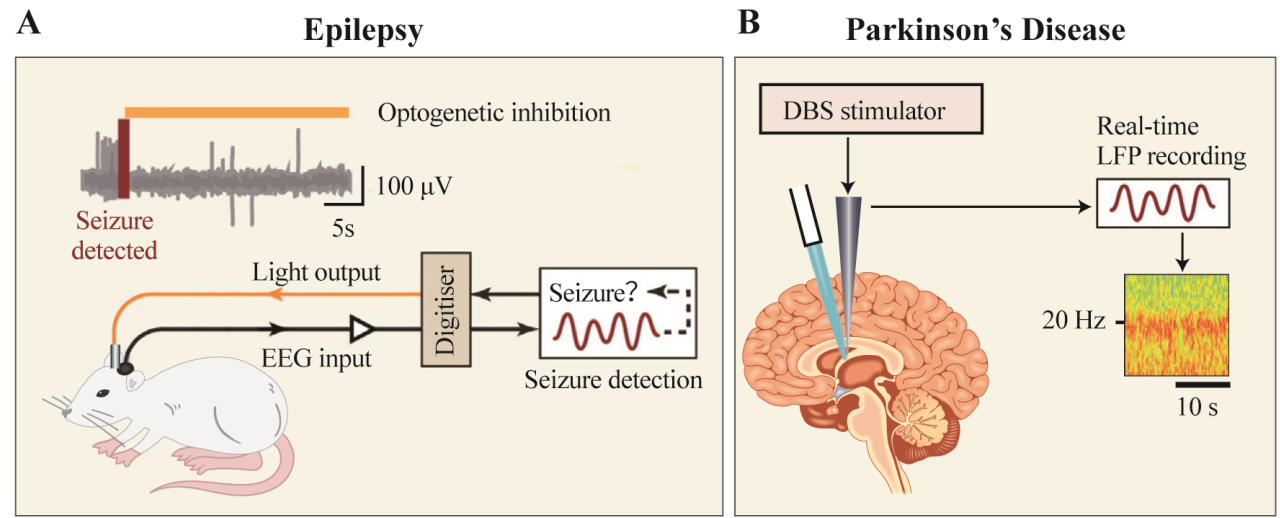

\section{Drug addiction}

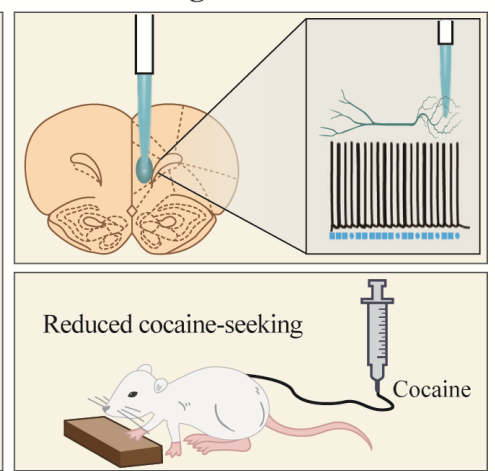

Figure 3 Application of optogenetics to disease treatment (revised from Rajasethupathy et al $2016^{59}$ ). (A) Preventing epilepsy by monitoring EEG signal combined with optogenetic techniques. (B) Relieving Parkinson's disease via optogenetics technology combined with deep brain stimulation technology. (C) Anti-addiction treatment by stimulating forebrain neuronal activity with optogenetical technology.

clozapine-N-oxide $(\mathrm{CNO})$ and then produce various reactions, either activating or inhibiting neuronal activity. The most widely used DREADDs receptors are Gq-DREADD and Gi-DREADD. ${ }^{61}$

In practice, we only need to clone the appropriate DREADDs receptor or Cre-dependent expression of the DREADDs receptor into the viral vector, and then express the virus in a specific brain region and then control the cell activity by administering CNO. Compared with optogenetics, the advantages of chemical genetics are as follows: first, fibre embedding is not necessary in DREADDs strategies, which makes it non-invasive and low cost; second, DREADDs can activate or inhibit cell activity for a long time because it does not depend on the influx and efflux of ions; third, CNO is an FDA-approved clinical drug analogue with prospects for drug development. The weakness of DREADDs technology is that the cell activity cannot be controlled with accuracy. Because of drug metabolism, it cannot provide us with time specificity and accuracy of effect. It is thus necessary to select appropriate technology according to the purpose of the experiment.

\section{Application of calcium ion imaging technology}

In the nervous system, calcium ions are the basis of cell signal production, and intracellular calcium concentration increases 10-100 times when neurons are activated. Therefore, researchers often observe neuronal activity by means of calcium ion imaging. With calcium imaging technology, the originally silent electrophysiological activity has become a spectacular image with vivid and colourful images. Researchers can track neuronal activity at the same time and analyse the significance.

Traditional calcium ion imaging techniques rely on chemical calcium indicators, such as BAPTA-based Oregon Green-1, Fura-2 and so forth. These indicators can specifically characterise $\mathrm{Ca}^{2+}$ concentrations but do not label specific cell types. Viral vectors were able to provide a better choice for such studies. We could clone proteins that indicate calcium ion concentrations into viruses, such as GCaMP, and then simultaneously observe $\mathrm{Ca}^{2+}$ concentrations and activities of large numbers of cells. So far, the most widely used GCaMP has been developed for many generations. ${ }^{62}$ Take GCaMP6f for example: it responded quickly but with weak intensity, and GCaMP6s were with high intensity but with slow response. Therefore, we need to select a suitable GCaMP proteins according to the needs in actual experiments.

Under the circumstances of combining specific functional viral vectors, we can also retrograde track and observe neuronal activity in a neuronal circuit or by recording neural activity across the whole brain through a blood-brain barrier viral vector. At the same time, with the development of calcium ion indicator proteins, a new calcium-sensitive protein, such as the red calcium-sensitive protein jRGECO1a, ${ }^{63}$ was constructed to simultaneously observe changes in two cell populations in the same brain region. With the induction of UV, the fluorescence of CaMPARI protein can be permanently changed from green to red, and neuronal activity can be recorded permanently as well. ${ }^{64}$

\section{APPLICATION OF VIRAL VECTOR IN THE CLINICAL THERAPY OF NEUROLOGICAL DISEASES \\ Viral vector-based gene therapy}

The engineered low immunogenic viral vector, which has good ability to carry genes, is very suitable for gene therapy. The current strategies for gene therapy include three categories: first, to replace the mutant gene with the correct gene by using a viral vector; second, to directly repair the wrong gene, such as CRISPR/Cas9, using gene editing technology; third, to perform the genetic modification of the cells with the lentivirus infection, and then transplanted the transformed cells back to the patient, by such as the chimeric antigen receptor T-cell immunotherapy (CAR-T) technique. 
AAV has become a preferred gene carrier for a large number of diseases, including neurological diseases, motor diseases and ophthalmology diseases, owing to its high infection efficiency and extremely low immunogenicity. To date, there are at least $110 \mathrm{AAV}$-related gene therapies in the world for clinical trials. Glybera ${ }^{65}$ was approved by the European Food and Drug Administration for the treatment of lipoprotein lipase deficiency in 2013. The Luxturna gene therapy for the treatment of hereditary retinal dystrophy ${ }^{66}$ was approved by the US FDA in 2017.

At the same time, lentivirus-based CAR-T therapy is also very popular around the world. More than 285 CAR-T-related therapies are in clinical trials. The FDA already approved Kymriah, ${ }^{67}$ an acute lymphoblastic leukaemia therapy in 2017. Treatment of relapsed or refractory children and young adult B cells with lentivirus-based CAR-T therapy kicked off the new gene therapy. In the future, with the deep understanding of neuroscience, the dream of using viral vectors to repair defective genes and to treat mental and neurological diseases could become a reality.

\section{Combination of clinical research and basic research in neuroscience}

With the rapid development of genome sequencing technology and high-throughput expression analytical technology, analysis of transcriptome, proteome, metabolome and non-coding genes such as microRNA, LncRNA and CircleRNA in clinical disease samples has become common. In this way, we can screen for characteristic genes related to mental illness, and further carry out two types of work: one is to look for diagnostic markers of diseases, which could assist in the diagnosis of diseases. The other is to investigate the underlying disease mechanisms, which could help with drug discovery.

Diagnostic markers for diseases are critical for the early prevention and early intervention of diseases. In addition to analysing free proteins, DNA or RNA in body fluid samples (blood, urine, lymph, cerebrospinal fluid, etc), the current study can also focus on detecting exosomes. Exosomes are vesicle-like bodies secreted by cells, which enclose large amounts of protein and nucleic acid substances, and some of them have been identified as disease markers. Usually, exosomes can be isolated and extracted from blood and urine samples, which lays a good foundation for non-invasive clinical diagnosis. In addition, exosomes can also be used as drug carriers due to their excellent membrane fusion properties.

Based on the abovementioned gene screening, we can also deeply investigate the molecular mechanisms of mental illness, including genetic factors, epigenetic factors and non-coding genetic factors. Here, we can quickly verify gene function in disease models, figure out the underlying mechanisms and screen drugs by viral vector-based gene manipulation. It is worth noting that the development of single-cell sequencing technology has brought new opportunities for the development of this field. Currently, we can use the patch clamp technique to absorb the contents of individual cells and scan their genomes, which helps to discover and manipulate new cell populations, or more accurately focus on specific types of cells. The activity and genetic components of individual cells can thus be recorded and identified. It is also one of the most important directions and future cornerstones for the development of viral vector application.

The development of new tools can also help with the development of neuroscience research with Chinese characteristics. In traditional Chinese culture and medicine, many important mechanisms of disease intervention are not clear, such as the physiological and biochemical mechanisms underlying acupuncture, massage and meditation. In addition, a variety of boxing styles in Chinese traditional martial arts have also been proven to improve the symptoms of depression or other mental illness, but the mechanism is still unclear. It is therefore difficult to conduct in-depth research using conventional approaches. In the future, with the help of viral vectors and a series of new technologies, we may bring new treatment ideas and methods, such as exploring the role of acupuncture through optogenetics. Chemical genetics might be employed to continuously activate motor neurons for treating depression. Chinese culture and Chinese medicine are treasures of scientific research. Understanding their mechanisms and making use of them in clinical practice are beneficial for physical health and disease rehabilitation.

\section{CONCLUSION}

Psychiatry is one of the disciplines most closely related to neuroscience. To carry out the National Brain Program, the development of therapeutic methods for mental or neurological diseases, such as early diagnosis and early detection of depression and autism, has received widespread attention from neuroscientists. The development of viral vectors and new technologies offers the opportunity for better research methods. Thus, understanding the basic principles and applications of viral vectors will be crucial for deciphering the pathologies of mental disease.

Acknowledgements This work was co-supported by National Natural Science Foundation of China (81571326, 81501153).

Contributors YW and JC completed the conception, data collection and classification of this article, and wrote and polished the full text. ZH conducted the drawing of the full-text chart. SY conducted the text collation and the writing of the gene therapy part. OP and FW improved the colour and provided suggestions for improvement and practical experience. PJ and JC conducted proof reading and revision of the full text.

Funding This work was co-supported by National Natural Science Foundation of China (81571326, 81501153).

Competing interests None declared.

Patient consent Not required.

Provenance and peer review Not commissioned; externally peer reviewed. 
Data sharing statement No additional data are available.

Open access This is an open access article distributed in accordance with the Creative Commons Attribution Non Commercial (CC BY-NC 4.0) license, which permits others to distribute, remix, adapt, build upon this work non-commercially, and license their derivative works on different terms, provided the original work is properly cited and the use is non-commercial. See:http://creativecommons.org/ licenses/by-nc/4.0

\section{REFERENCES}

1 Herculano-Houzel S. The human brain in numbers: a linearly scaledup primate brain. Front Hum Neurosci 2009;3:31.

2 Azevedo FA, Carvalho LR, Grinberg LT, et al. Equal numbers of neuronal and nonneuronal cells make the human brain an isometrically scaled-up primate brain. J Comp Neurol 2009;513:532-41.

3 Herculano-Houzel S. The remarkable, yet not extraordinary, human brain as a scaled-up primate brain and its associated cost. Proc Natl Acad Sci U S A 2012;109(Suppl 1):10661-8.

4 Fonseca-Azevedo K, Herculano-Houzel S. Metabolic constraint imposes tradeoff between body size and number of brain neurons in human evolution. Proc Natl Acad Sci U S A 2012;109:18571-6.

5 Carter BJ. Adeno-associated virus and the development of adeno-associated virus vectors: a historical perspective. Mol Ther 2004;10:981-9.

6 Kobayashi K, Kato S, Kobayashi K. Genetic manipulation of specific neural circuits by use of a viral vector system. J Neural Transm 2018;125:67-75.

7 Diester I, Kaufman MT, Mogri M, et al. An optogenetic toolbox designed for primates. Nat Neurosci 2011;14:387-97.

8 Alivisatos AP, Chun M, Church GM, et al. The brain activity map project and the challenge of functional connectomics. Neuron 2012;74:970-4.

9 Poo MM, Du JL, Ip NY, et al. China Brain Project: Basic neuroscience, brain diseases, and brain-inspired computing. Neuron 2016;92:591-6.

10 Shields BC, Kahuno E, Kim C, et al. Deconstructing behavioral neuropharmacology with cellular specificity. Science 2017;356:eaaj2161.

11 Jackson DA, Symons RH, Berg P. Biochemical method for inserting new genetic information into DNA of Simian Virus 40: circular SV40 DNA molecules containing lambda phage genes and the galactose operon of Escherichia coli. Proc Natl Acad Sci U S A 1972;69:2904-9.

12 Ojala DS, Amara DP, Schaffer DV. Adeno-associated virus vectors and neurological gene therapy. Neuroscientist 2015;21:84-98.

13 Hocquemiller M, Giersch L, Audrain M, et al. Adeno-associated virus-based gene therapy for CNS diseases. Hum Gene Ther 2016;27:478-96.

14 Wang W, Wildes CP, Pattarabanjird T, et al. A light- and calciumgated transcription factor for imaging and manipulating activated neurons. Nat Biotechnol 2017;35:864-71.

15 Lee $\mathrm{D}$, Hyun JH, Jung $\mathrm{K}$, et al. A calcium- and light-gated switch to induce gene expression in activated neurons. Nat Biotechnol 2017;35:858-63.

16 Liu X, Ramirez S, Pang PT, et al. Optogenetic stimulation of a hippocampal engram activates fear memory recall. Nature 2012;484:381-5

17 Tsien RY. The green fluorescent protein. Annu Rev Biochem 1998;67:509-44.

18 Shaner NC, Lambert GG, Chammas A, et al. A bright monomeric green fluorescent protein derived from Branchiostoma lanceolatum. Nat Methods 2013;10:407-9.

19 Bindels DS, Haarbosch L, van Weeren L, et al. mScarlet: a bright monomeric red fluorescent protein for cellular imaging. Nat Methods 2017;14:53-6.

20 Okuyama T, Kitamura T, Roy DS, et al. Ventral CA1 neurons store social memory. Science 2016;353:1536-41.

$21 \mathrm{Kim}$ J, Zhao T, Petralia RS, et al. mGRASP enables mapping mammalian synaptic connectivity with light microscopy. Nat Methods 2011:9:96-102.

22 Feinberg $\mathrm{EH}$, Vanhoven MK, Bendesky A, et al. Gfp reconstitution across synaptic partners (grasp) defines cell contacts and synapses in living nervous systems. Neuron 2008;57:353-63.

23 Feng L, Kwon O, Lee B, et al. Using mammalian GFP reconstitution across synaptic partners (mGRASP) to map synaptic connectivity in the mouse brain. Nat Protoc 2014;9:2425-37.
24 Zhu LJ, Li TY, Luo CX, et al. CAPON-nNOS coupling can serve as a target for developing new anxiolytics. Nat Med 2014;20:1050-4

$25 \mathrm{Li} \mathrm{Y,} \mathrm{Xu} \mathrm{J,} \mathrm{Liu} \mathrm{Y,} \mathrm{et} \mathrm{al.} \mathrm{A} \mathrm{distinct} \mathrm{entorhinal} \mathrm{cortex} \mathrm{to} \mathrm{hippocampal}$ CA1 direct circuit for olfactory associative learning. Nat Neurosci 2017;20:559-70.

26 Liu Y, Miao Q, Yuan J, et al. Ascl1 converts dorsal midbrain astrocytes into functional neurons in vivo. J Neurosci 2015;35:9336-55.

27 Tsien JZ. Cre-lox neurogenetics: 20 years of versatile applications in brain research and counting.... Front Genet 2016;7:19.

28 Fenno LE, Mattis J, Ramakrishnan C, et al. Targeting cells with single vectors using multiple-feature Boolean logic. Nat Methods 2014;11:763-72.

29 Atasoy D, Aponte Y, Su HH, et al. A FLEX switch targets Channelrhodopsin-2 to multiple cell types for imaging and longrange circuit mapping. J Neurosci 2008;28:7025-30.

$30 \mathrm{Du} \mathrm{H}$, Deng W, Aimone JB, et al. Dopaminergic inputs in the dentate gyrus direct the choice of memory encoding. Proc Natl Acad Sci U S A 2016;113:E5501-E5510.

31 Ma J, Zhang J, Hou WW, et al. Early treatment of minocycline alleviates white matter and cognitive impairments after chronic cerebral hypoperfusion. Sci Rep 2015;5:12079.

32 Wang X, Tsai JW, LaMonica B, et al. A new subtype of progenitor cell in the mouse embryonic neocortex. Nat Neurosci 2011;14:555-61.

33 Zheng J, Jiang YY, Xu LC, et al. Adult hippocampal neurogenesis along the dorsoventral axis contributes differentially to environmental enrichment combined with voluntary exercise in alleviating chronic inflammatory pain in mice. $J$ Neurosci 2017;37:4145-57.

34 Choi JH, Sim SE, Kim Jl, et al. Interregional synaptic maps among engram cells underlie memory formation. Science 2018;360:430-5.

35 Tkatch T, Greotti E, Baranauskas G, et al. Optogenetic control of mitochondrial metabolism and $\mathrm{Ca}^{2+}$ signaling by mitochondriatargeted opsins. Proc Natl Acad Sci U S A 2017;114:E5167-E76.

36 Mardinly AR, Oldenburg IA, Pégard NC, et al. Precise multimodal optical control of neural ensemble activity. Nat Neurosci 2018;21:881-93.

37 Sinnen BL, Bowen AB, Forte JS, et al. Optogenetic control of synaptic composition and function. Neuron 2017;93:646-60.

38 Ding $\mathrm{X}$, Liu S, Tian M, et al. Activity-induced histone modifications govern Neurexin-1 mRNA splicing and memory preservation. Nat Neurosci 2017;20:690-9.

39 Kristensson K, Svennerholm B, Vahlne A, et al. Virus-induced demyelination in herpes simplex virus-infected mice. J Neurol Sci 1982;53:205-16.

40 Mu D, Deng J, Liu KF, et al. A central neural circuit for itch sensation. Science 2017;357:695-9.

41 Tervo DG, Hwang BY, Viswanathan S, et al. A designer AAV variant permits efficient retrograde access to projection neurons. Neuron 2016:92:372-82.

42 Zingg B, Chou XL, Zhang ZG, et al. AAV-mediated anterograde transsynaptic tagging: mapping corticocollicular input-defined neural pathways for defense behaviors. Neuron 2017:93:33-47.

$43 \mathrm{Xu} \mathrm{W}$, Südhof TC. A neural circuit for memory specificity and generalization. Science 2013;339:1290-5.

44 Chan KY, Jang MJ, Yoo BB, et al. Engineered AAVs for efficient noninvasive gene delivery to the central and peripheral nervous systems. Nat Neurosci 2017;20:1172-9.

45 Kaspar BK, Vissel B, Bengoechea T, et al. Adeno-associated virus effectively mediates conditional gene modification in the brain. Proc Natl Acad Sci U S A 2002;99:2320-5.

46 Ahmed BY, Chakravarthy S, Eggers R, et al. Efficient delivery of Crerecombinase to neurons in vivo and stable transduction of neurons using adeno-associated and lentiviral vectors. BMC Neurosci 2004;5:4.

47 Yao XH, Wang M, He XN, et al. Electrical coupling regulates layer 1 interneuron microcircuit formation in the neocortex. Nat Commun 2016;7:12229.

48 Cohen J. The birth of CRISPR Inc. Science 2017;355:680-4.

49 Gaj T, Gersbach CA, Barbas CF. ZFN, TALEN, and CRISPR/ Cas-based methods for genome engineering. Trends Biotechnol 2013;31:397-405.

50 Swiech L, Heidenreich M, Banerjee A, et al. In vivo interrogation of gene function in the mammalian brain using CRISPR-Cas9. Nat Biotechnol 2015;33:102-6.

51 Platt RJ, Chen S, Zhou Y, et al. CRISPR-Cas9 knockin mice for genome editing and cancer modeling. Cell 2014;159:440-55.

52 Liao HK, Hatanaka F, Araoka T, et al. In Vivo target gene activation via CRISPR/Cas9-mediated trans-epigenetic modulation. Cell 2017;171:1495-507. 
53 Du D, Qi LS. Crispr technology for genome activation and repression in mammalian cells. Cold Spring Harb Protoc 2016;2016:pdb prot090175.

54 Yizhar O, Fenno LE, Davidson TJ, et al. Optogenetics in neural systems. Neuron 2011;71:9-34.

55 Gunaydin LA, Yizhar O, Berndt A, et al. Ultrafast optogenetic control. Nat Neurosci 2010;13:387-92.

56 Zhou T, Zhu H, Fan Z, et al. History of winning remodels thalamo-PFC circuit to reinforce social dominance. Science 2017;357:162-8.

57 Nussinovitch U, Gepstein L. Optogenetics for in vivo cardiac pacing and resynchronization therapies. Nat Biotechnol 2015;33:750-4.

58 Shao J, Xue S, Yu G, et al. Smartphone-controlled optogenetically engineered cells enable semiautomatic glucose homeostasis in diabetic mice. Sci Trans/ Med 2017:9:eaal2298.

59 Rajasethupathy P, Ferenczi E, Deisseroth K. Targeting neural circuits. Cell 2016;165:524-34.

60 Creed M, Pascoli VJ, Lüscher C. Addiction therapy. Refining deep brain stimulation to emulate optogenetic treatment of synaptic pathology. Science 2015;347:659-64.
61 Sternson SM, Roth BL. Chemogenetic tools to interrogate brain functions. Annu Rev Neurosci 2014;37:387-407.

62 Chen TW, Wardill TJ, Sun Y, et al. Ultrasensitive fluorescent proteins for imaging neuronal activity. Nature 2013;499:295-300.

63 Meng C, Zhou J, Papaneri A, et al. Spectrally resolved fiber photometry for multi-component analysis of brain circuits. Neuron 2018;98:707-17.

64 Fosque BF, Sun Y, Dana H, et al. Neural circuits. Labeling of active neural circuits in vivo with designed calcium integrators. Science 2015;347:755-60.

65 Ylä-Herttuala S. Endgame: glybera finally recommended for approval as the first gene therapy drug in the European Union. Mol Ther 2012;20:1831-2.

66 The Medical Letter. Voretigene neparvovec-rzyl (Luxturna) for inherited retinal dystrophy. Med Lett Drugs Ther 2018;60:53-5.

67 Morrow T. Novartis's Kymriah: harnessing immune system comes with worry about reining in costs. Manag Care 2017;26:28-30.

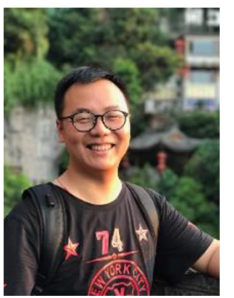

Yao Wang obtained the bachelor degree of Science from Shandong Agriculture University (SDAU) in 2007, and a Master degree of Genomics and Ph.D. of Neuroscience from East China Normal University (ECNU) in 2010 and 2016, respectively. From Sep. 2010 to Feb. 2017, Yao worked at Key Laboratory of Brain Functional Genomics, Ministry of Education of ECNU as an assistant professor. After that, he joined OBiO Technology (Shanghai) Corp., Ltd. to be the director of marketing department. Now he is in charge of the application of viral vector in Neuroscience, Metabolism and Oncology. His research interests include the synaptic plasticity of learning and memory. He also interested in mechanisms of neuropathic pain and mental disease, such as depression and autism. 